\title{
Chromatic Graphs, Ramsey Numbers and the Flexible Atom Conjecture
}

\author{
Jeremy F. Alm, Roger D. Maddux, Jacob Manske \\ Department of Mathematics \\ University of Dallas, Irving, TX, USA \\ alm@udallas .edu \\ Department of Mathematics \\ Iowa State University, Ames, IA, USA \\ maddux@iastate.edu \\ Department of Mathematics \\ Iowa State University, Ames, IA, USA \\ jmanske@iastate.edu
}

Submitted: Nov 13, 2007; Accepted: Mar 17, 2008; Published: Mar 27, 2008

Mathematics Subject Classification: 05D40, 03G15

\begin{abstract}
Let $K_{N}$ denote the complete graph on $N$ vertices with vertex set $V=V\left(K_{N}\right)$ and edge set $E=E\left(K_{N}\right)$. For $x, y \in V$, let $x y$ denote the edge between the two vertices $x$ and $y$. Let $L$ be any finite set and $\mathcal{M} \subseteq L^{3}$. Let $c: E \rightarrow L$. Let $[n]$ denote the integer set $\{1,2, \ldots, n\}$.

For $x, y, z \in V$, let $c(x y z)$ denote the ordered triple $(c(x y), c(y z), c(x z))$. We say that $c$ is good with respect to $\mathcal{M}$ if the following conditions obtain:

(i) $\forall x, y \in V$ and $\forall(c(x y), j, k) \in \mathcal{M}, \exists z \in V$ such that $c(x y z)=(c(x y), j, k)$;

(ii) $\forall x, y, z \in V, c(x y z) \in \mathcal{M}$; and

(iii) $\forall x \in V \forall \ell \in L \exists y \in V$ such that $c(x y)=\ell$.

We investigate particular subsets $\mathcal{M} \subseteq L^{3}$ and those edge colorings of $K_{N}$ which are good with respect to these subsets $\mathcal{M}$. We also remark on the connections of these subsets and colorings to projective planes, Ramsey theory, and representations of relation algebras. In particular, we prove a special case of the flexible atom conjecture.
\end{abstract}

\section{Motivation and background}

Let $K_{N}$ denote the complete graph on $N$ vertices with vertex set $V=V\left(K_{N}\right)$ and edge set $E=E\left(K_{N}\right)$. For $x, y \in V$, let $x y$ denote the edge between the two vertices $x$ and 
$y$. Let $L$ be any finite set and $\mathcal{M} \subseteq L^{3}$. Let $c: E \rightarrow L$. Let $[n]$ denote the integer set $\{1,2, \ldots, n\}$.

For $x, y, z \in V$, let $c(x y z)$ denote the ordered triple $(c(x y), c(y z), c(x z))$. We say that $c$ is good with respect to $\mathcal{M}$ if the following conditions obtain:

(i) $\forall x, y \in V$ and $\forall(c(x y), j, k) \in \mathcal{M}, \exists z \in V$ such that $c(x y z)=(c(x y), j, k)$;

(ii) $\forall x, y, z \in V, c(x y z) \in \mathcal{M}$; and

(iii) $\forall x \in V \forall \ell \in L \exists y \in V$ such that $c(x y)=\ell$.

If $K=K_{N}$ has a coloring $c$ which is good with respect to $\mathcal{M}$, then we say that $K$ realizes $\mathcal{M}$ (or that $\mathcal{M}$ is realizable).

If we take $R_{\alpha}=\{(x, y): c(x y)=\alpha\}$, and let $\mid$ stand for ordinary composition of binary relations, ie. $R_{\alpha} \mid R_{\beta}:=\left\{(x, z): \exists y(x, y) \in R_{\alpha},(y, z) \in R_{\beta}\right\}$, then conditions (i) and (ii) imply

$$
\left(R_{\alpha} \mid R_{\beta}\right) \cap R_{\gamma} \neq \emptyset \Longrightarrow R_{\gamma} \subseteq R_{\alpha} \mid R_{\beta} .
$$

Conditions (i) - (iii) are given in [1] where the author calls a coloring on $K_{N}$ that realizes some $\mathcal{M}$ a symmetric color scheme. It is proved in [2] that if $\mathcal{M}$ is a set of triples that is closed under permutation such that there is at least one $\alpha \in L$ such that for all $\beta, \gamma \in L$, $(\alpha, \beta, \gamma) \in \mathcal{M}$, then $\mathcal{M}$ is realized by a coloring on $K_{\omega}$, the complete graph on countably many vertices. Any such color $\alpha$ is called a flexible color, since it can participate in any triple.

Conditions (i) - (iii) may seem quite stringent, but in fact these conditions are satisfied in many natural situations. Recall the notation for the Ramsey numbers; that is, $R\left(k_{1}, k_{2}, \ldots, k_{\ell}\right)$ is the minimum integer $n$ such that in any $\ell$-coloring of the edges of $K_{n}$ there is a monochromatic complete graph on $k_{j}$ vertices in color $j$ for some $j$. In particular, the coloring of $K_{5}$ which shows $R(3,3) \geq 6$ satisfies (i) - (iii), as does the coloring of $K_{8}$ which shows $R(4,3) \geq 9$, the colorings of $K_{16}$ that show $R(3,3,3) \geq 17$, both "twisted" and "untwisted", and the coloring of $K_{29}$ given in [7] and [4] that shows that $R(4,3,3) \geq 30$. In fact, the coloring of $K_{5}$ without monochromatic triangles is a realization of $\mathcal{M}_{0}=\{(r, b, b),(b, r, b),(b, b, r),(r, r, b),(r, b, r),(b, r, r)\}$. The coloring of $K_{8}$ mentioned above is a realization of $\mathcal{M}=\mathcal{M}_{0} \cup\{(r, r, r)\}$; the col orings of $K_{16}$ are realizations of $\mathcal{M}=\{r, b, g\}^{3} \backslash\{(r, r, r),(b, b, b),(g, g, g)\}$; the coloring of $K_{29}$ is a realization of $\mathcal{M}=\{r, b, g\}^{3} \backslash\{(b, b, b),(g, g, g)\}$.

In [1], Comer introduces the number $r(k)$ which is the largest $N$ such that there is a coloring on $K_{N}$ that realizes

$$
\mathcal{M}=\left\{r_{1}, \ldots, r_{k}\right\}^{3} \backslash\left\{\left(r_{i}, r_{i}, r_{i}\right): i \in[k]\right\} .
$$

Clearly, $r(k) \leq R(\overbrace{3,3, \ldots, 3}^{k \text { times }})-1$; equality holds for $k=2$ and $k=3$. An interesting open problem is whether equality holds for all values of $k$. 
Realizations of color schemes arise in connection with projective planes as well. Let $L=\left\{r_{1}, \ldots, r_{\ell}\right\}$, and let

$$
\mathcal{M}_{\ell}=\left\{\left(r_{i}, r_{j}, r_{k}\right):|\{i, j, k\}| \in\{1,3\}\right\} .
$$

Lyndon proved in [5] that $\mathcal{M}_{\ell}$ is realizable in some complete graph if and only if there exists a projective plane of order $\ell-1$, for $\ell>2$. This result has been extremely important in the theory of relation algebras.

In [3], Maddux, Jipsen and Tuza show that for $\mathcal{M}=L^{3}, K_{N}$ realizes $\mathcal{M}$ for arbitrarily large finite $N$. In the case when $\mathcal{M}=L^{3}$, every color in $L$ is a flexible color.

\section{The Main Result}

The principal result of this paper is that $\mathcal{M}_{n}$ is realizable in $K_{N}$ for some $N<\omega$, where $L=\left\{r, b_{1}, \ldots, b_{n}\right\}$ and

$$
\mathcal{M}_{n}:=\left\{(r, r, r),\left(r, r, b_{i}\right),\left(r, b_{i}, r\right),\left(b_{i}, r, r\right),\left(r, b_{i}, b_{j}\right),\left(b_{i}, r, b_{j}\right),\left(b_{i}, b_{j}, r\right): i, j \in[n]\right\} .
$$

(Observe that $\mathcal{M}_{n}=\left\{r, b_{1}, \ldots, b_{n}\right\}^{3} \backslash\left\{b_{1}, \ldots, b_{n}\right\}^{3}$.) This is a special case of a problem that has come to be known as the flexible atom conjecture. This problem originates in relation algebra; an explanation of the conjecture in this context can be found in [6].

Theorem 1. $\forall n \geq 1 \exists r=r(n)$ such that $\forall k>r, K_{N}$ realizes $\mathcal{M}_{n}$ for $N=\left(\begin{array}{c}3 k-4 \\ k\end{array}\right)$.

The proof will proceed as follows. First we will construct realizations of $M_{1}$ in $K_{N}$ for arbitrarily large $N$. These colorings of $K_{N}$ will exhibit quite a lot of redundancy; in particular, for any given edge $x y \in E$ and triple $(c(x y), j, k) \in \mathcal{M}_{1}$, there exist many vertices $z$ such that $c(x y z)=(c(x y), j, k)$, while condition (i) only requires that there be one such vertex. The graph $K_{N}$, which is colored in colors $r$ and $b$, can then be recolored by assigning edges colored $b$ to a color from $\left\{b_{1}, \ldots, b_{n}\right\}$ uniformly at random. The probability that this recoloring realizes $\mathcal{M}_{n}$ is shown to be nonzero for sufficiently large $N$.

Note that $r$ is a flexible color in $\mathcal{M}_{n}$. In the case that a flexible color is present, it is not hard to see that condition (iii) is automatically satisfied whenever (i) and (ii) are, and so we make no further mention of it.

\section{Proof of theorem 1}

Let $k \in \mathbb{N}$ and let $[3 k-4]^{k}$ denote the collection of $k$-subsets of $[3 k-4]$. Let $G$ be the complete graph with vertex set $V=[3 k-4]^{k}$.

Lemma 1. $G$ realizes $\mathcal{M}_{1}$ for any $k \geq 3$. 
Proof of lemma 1. Define an edge coloring $c: E(G) \rightarrow\{r, b\}$ by

$$
c(x y)= \begin{cases}b, & \text { if } 0 \leq|x \cap y| \leq 1 \\ r, & \text { otherwise }\end{cases}
$$

Let $E_{r}=\{x y \in E(G): c(x y)=r\}$ and $E_{b}=\{x y \in E(G): c(x y)=b\}$. The following five claims establish that $c$ satisfies condition (i) for $\mathcal{M}_{1}$.

Let $x y \in E_{r}$. Since $|x \cap y| \geq 2,|x \cup y| \leq 2 k-2$.

Claim 1. $\exists z \in V$ such that $c(x y z)=(r, r, r)$.

Let $\overline{(x \cup y)}$ denote $[3 k-4] \backslash(x \cup y)$ and let $\ell$ be any subset of $\overline{(x \cup y)}$ with $k-|x \cap y|$ elements. Set $z=\ell \cup(x \cap y)$. We have $|x \cap z| \geq 2$ and $|y \cap z| \geq 2$, so $c(x y z)=(r, r, r)$ and claim 1 is true.

Claim 2. $\exists z \in V$ such that $c(x y z)=(r, r, b)$.

Let $a_{1} \in y \backslash x, a_{2} \in x \cap y$, and $\ell$ be any $(k-2)$-subset of $\overline{(x \cup y)}$. Set $z=\ell \cup\left\{a_{1}, a_{2}\right\}$. We have $|x \cap z|=1$ and $|y \cap z|=2$, so $c(x y z)=(r, r, b)$ and claim 2 is true.

Claim 3. $\exists z \in V$ such that $c(x y z)=(r, b, b)$.

Let $a_{1} \in x \backslash y, a_{2} \in y \backslash x$. Let $\ell$ be as in the the proof of claim 2. Set $z=\ell \cup\left\{a_{1}, a_{2}\right\}$. We have $|x \cap z|=|y \cap z|=1$, so $c(x y z)=(r, b, b)$ and claim 3 is true.

Now let $x y \in E_{b}$. Since $|x \cap y| \leq 1,|x \cup y| \geq k-3$.

Claim 4. $\exists z \in V$ such that $c(x y z)=(b, r, r)$.

If $k=3$, then $|x \cap y|=1$, so we can pick $z$ to be the 3 -subset consisting of $x \cap y$, one point from $x \backslash y$ and one point in $y \backslash x$. For $k \geq 4$, let $\ell_{1}$ be any 2-subset of $x \backslash y, \ell_{2}$ be any 2-subset of $y \backslash x$, and $\ell_{3}$ be any $(k-4)$-subset of $\overline{(x \cup y)}$. Set $z=\ell_{1} \cup \ell_{2} \cup \ell_{3}$. We have $|x \cap z|=2$ and $|y \cap z|=2$, so $c(x y z)=(b, r, r)$ and claim 4 is true.

Claim 5. $\exists z \in V$ such that $c(x y z)=(b, b, r)$.

If $k=3$, then $|x \cap y|=1$, so we can pick $z$ to be the 3-subset consisting of $y \backslash x$ together with one point from $x \backslash y$. For $k \geq 4$, let $\ell_{1}$ be any 3-subset of $x \backslash y$ and $a \in y \backslash x$. Let $\ell_{3}$ be as in the proof of claim 4. Set $z=\ell_{1} \cup\{a\} \cup \ell_{3}$. We have $|x \cap z| \geq 2$ and $|y \cap z|=1$, so $c(x y z)=(b, b, r)$ and claim 5 is true.

Observe that claims 1-5 imply that $c$ satisfies condition (i) for $\mathcal{M}_{1}$. It remains to show that $c$ satisfies condition (ii) for $\mathcal{M}_{1}$, which we show in claim 6 below.

Claim 6. $\forall x, y, z \in V, c(x y z) \in \mathcal{M}_{1}$.

By way of contradiction, suppose $\exists x, y, z \in V$ with $c(x y z)=(b, b, b)$. Since $|x \cup y \cup z| \leq$ $3 k-4$, the pigeonhole principle implies that one of $|x \cap y|,|x \cap z|$, or $|y \cap z|$ is greater than or equal to 2 , a contradiction.

Observe that claims 1-6 imply that $c$ is good with respect to $\mathcal{M}_{1}$, and thus $G$ realizes $\mathcal{M}_{1}$ 
Let $n \geq 2$ and let $E_{r}$ and $E_{b}$ be as in the proof of lemma 1 . Let $\mathcal{S}$ be the set of all $n$-ary sequences of length $m=\left|E_{b}\right|$ taking digits from $[n]$. Choose a sequence $s$ from $\mathcal{S}$ at random. Enumerate the edges of $E_{b}: e_{1}, \ldots, e_{m}$. Let $s(j) \in[n]$ denote the $j^{\text {th }}$ position of the sequence $s$. Define a partition of $E_{b}$ into $n$ (possibly empty) parts $E_{b_{1}}, \ldots, E_{b_{n}}$ as follows:

$$
E_{b_{i}}=\left\{e_{j}: s(j)=i\right\}, i \in[n]
$$

Define a new edge coloring of $G$ given by

$$
c^{\prime}(x y)= \begin{cases}b_{i} & \text { if } x y \in E_{b_{i}}, \\ r & \text { if } x y \in E_{r} .\end{cases}
$$

It is not hard to see that the probability that a given edge has color $i$ is $1 / n$; and furthermore that, given two distinct edges, the assignment of their colors is independent.

We claim that for sufficiently large $k, c^{\prime}$ is good with respect to $\mathcal{M}_{n}$, and thus $G$ realizes $\mathcal{M}_{n}$; for this reason, we assume that $k \geq 4$. Since $c$ satisfies condition (ii) for $\mathcal{M}_{1}$, it is easy to see $c^{\prime}$ satisfies condition (ii) for $\mathcal{M}_{n}$. We show that the probability that $c^{\prime}$ does not satisfy condition (i) for $\mathcal{M}_{n}$ is less than 1.

Claim 7. The probability $P_{1}$ that given $x y \in E_{r}, \exists i, j \in[n]$ such that $\forall z \in V c^{\prime}(x y z) \neq$ $\left(r, b_{i}, b_{j}\right)$ is bounded from above by $n^{2}\left(1-1 / n^{2}\right)^{(k-2)^{2}}$.

Proof of claim \%. Let $Z:=\{z \in V: c(x y z)=(r, b, b)\}$. For fixed $i, j \in[n]$ and $z \in Z$, the probability

$$
\left(x z \in E_{b_{i}}\right) \wedge\left(y z \in E_{b_{j}}\right)
$$

is $1 / n^{2}$, so the probability

$$
\left(x z \notin E_{b_{i}}\right) \vee\left(y z \notin E_{b_{j}}\right)
$$

is $1-1 / n^{2}$. Considering all $z \in Z$, we have that the probability

$$
\bigwedge_{z \in Z}\left[\left(x z \notin E_{b_{i}}\right) \vee\left(y z \notin E_{b_{j}}\right)\right]
$$

is $\left(1-1 / n^{2}\right)^{|Z|}$. Summing over all $n^{2}$ combinations of $i$ and $j$, we arrive at

$$
P_{1}=n^{2}\left(1-1 / n^{2}\right)^{|Z|} .
$$

For an upper bound on $P_{1}$ we compute a lower bound on $|Z|$. Since we seek a lower bound, we may assume $|x \cap y|=2$. Note that $|\overline{(x \cup y)}|=k-2$. Let $a_{x} \in x \backslash y$ and $a_{y} \in y \backslash x$. If $z=\overline{(x \cup y)} \cup\left\{a_{x}, a_{y}\right\}$, then $z \in Z$. Since there are $(k-2)^{2}$ distinct $z$ of this form, $(k-2)^{2} \leq|Z|$. This fact together with $(1)$ gives $P_{1} \leq n^{2}\left(1-1 / n^{2}\right)^{(k-2)^{2}}$, as desired.

Claim 8. The probability $P_{2}$ that given $x y \in E_{r}, \exists j \in[n]$ such that $\forall z \in V c^{\prime}(x y z) \neq$ $\left(r, r, b_{j}\right)$ is bounded from above by $n(1-1 / n)^{\left(\begin{array}{c}k-2 \\ 2\end{array}\right)}$. 
Proof of claim 8. Let $Z:=\{z \in V: c(x y z)=(r, r, b)\}$. For fixed $j \in[n]$ and $z \in Z$, the probability

$$
\left(x z \in E_{b_{j}}\right) \wedge\left(y z \in E_{r}\right)=\left(x z \in E_{b_{j}}\right)
$$

is $1 / n$, so the probability

$$
\left(x z \notin E_{b_{j}}\right)
$$

is $1-1 / n$. Considering all $z \in Z$, we have that the probability

$$
\bigwedge_{z \in Z}\left(x z \notin E_{b_{j}}\right)
$$

is $(1-1 / n)^{|Z|}$. Summing over all $j$, we arrive at

$$
P_{2}=n(1-1 / n)^{|Z|} \text {. }
$$

For an upper bound on $P_{2}$, we compute a lower bound on $|Z|$. As in claim 7, we may assume $|x \cap y|=2$ so $|\overline{(x \cup y)}|=k-2$. Let $\ell$ be any 2-subset of $\overline{(x \cup y)}$. If $z=(y \backslash x) \cup \ell$, then $z \in Z$. Since there are $\left(\begin{array}{c}k-2 \\ 2\end{array}\right)$ distinct $z$ of this form, $\left(\begin{array}{c}k-2 \\ 2\end{array}\right) \leq|Z|$. This fact together with $(2)$ gives $P_{2} \leq n(1-1 / n)^{\left(\begin{array}{c}k-2 \\ 2\end{array}\right)}$, as desired.

Claim 9. The probability $P_{3}$ that given $i \in[n]$ and $x y \in E_{b_{i}}, \exists j \in[n]$ such that $\forall z \in V$, $c^{\prime}(x y z) \neq\left(b_{i}, r, b_{j}\right)$ is bounded from above by $n(1-1 / n)^{\left(\begin{array}{c}k \\ 4\end{array}\right)}$.

Proof of claim 9. Fix $i \in[n]$ and $x y \in E_{b_{i}}$. Let

$$
Z:=\{z \in V: c(y z)=r \text { and } c(x z)=b\} .
$$

For $j \in[n]$, the probability that $x z \in E_{b_{j}}$ is $1 / n$, so the probability that $x z \notin E_{b_{j}}$ is $1-1 / n$. Continuing as in claim 8 , we have

$$
P_{3}=n(1-1 / n)^{|Z|} .
$$

Again, we seek a lower bound for $|Z|$, so we may assume $|x \cap y|=0$. Note that $|\overline{(x \cup y)}|=k-4$. Let $\ell$ be any 4-subset of $y$. If $z=\overline{(x \cup y)} \cup \ell$, then $z \in Z$. Since there are $\left(\begin{array}{l}k \\ 4\end{array}\right)$ distinct $z$ of this form, $\left(\begin{array}{l}k \\ 4\end{array}\right) \leq|Z|$. This fact together with (3) gives $P_{3} \leq n(1-1 / n)^{\left(\begin{array}{c}k \\ 4\end{array}\right)}$.

Observe that $\forall \ell, P_{1} \geq P_{\ell}$. Hence, we can use the upper bound in claim 7 for $P_{1}$ as an upper bound for the probability that condition (i) does not obtain for given $x y \in E$. Since $G$ has less than $\left(\begin{array}{c}3 k-4 \\ k\end{array}\right)^{2}$ edges, an upper bound for the probability $P$ that $c^{\prime}$ fails to satisfy condition (i) for $\mathcal{M}_{n}$ is

$$
P \leq \sum_{e \in E} P_{1} \leq\left(\begin{array}{c}
3 k-4 \\
k
\end{array}\right)^{2} P_{1} \leq\left(\begin{array}{c}
3 k-4 \\
k
\end{array}\right)^{2} n^{2}\left(1-\frac{1}{n^{2}}\right)^{(k-2)^{2}}
$$


Next, we show that the right hand side of the expression in (4) can be made less than 1 by choosing $k$ large enough. Since $1-x \leq e^{-x}$ for all $x$, we have

$$
\begin{aligned}
\left(\begin{array}{c}
3 k-4 \\
k
\end{array}\right)^{2} n^{2}\left(1-\frac{1}{n^{2}}\right)^{(k-2)^{2}} & \leq\left(\begin{array}{c}
3 k-4 \\
k
\end{array}\right)^{2} n^{2}\left(e^{-(k-2)^{2} / n^{2}}\right) \\
& \leq\left(2^{3 k-4}\right)^{2} n^{2}\left(e^{-(k-2)^{2} / n^{2}}\right) \\
& \leq 2^{6 k} n^{2}\left(e^{-(k-2)^{2} / n^{2}}\right)
\end{aligned}
$$

Note that the expression in (5) is less than 1 if and only if $\log \left[2^{6 k} n^{2}\left(e^{-(k-2)^{2} / n^{2}}\right)\right]<0$, which holds just in case

$$
6 k \log 2+2 \log n-\frac{(k-2)^{2}}{n^{2}}<0 .
$$

To ensure that the inequality in (6) will hold, we first assume that $k=c n^{2}$ for some $c \in \mathbb{R}$ and realize the above as a quadratic polynomial in $c$. Since the coefficient of $c^{2}$ is negative, the function is concave down. By finding the zeros of this polynomial in terms of $n$ and then maximizing (over $n$ ) the greatest of them, we can find the $c$ which will guarantee the inequality in (6). For $n \geq 2$, it is sufficient to take $c \geq 5.2$.

For such $k$, we have that $P<1$, so there exists an edge coloring $c: E(G) \rightarrow$ $\left\{r, b_{1}, \ldots, b_{n}\right\}$ which is good with respect to $\mathcal{M}_{n}$. Hence, $G$ realizes $\mathcal{M}_{n}$ and the proof of theorem 1 is complete.

Corollary 1. Any finite integral symmetric relation algebra with one flexible atom and with all (mandatory) diversity cycles involving the flexible atom is representable on arbitrarily large finite sets.

It is possible to obtain Corollary 1 with "symmetric" deleted in the following way. We alter the proof of Theorem 1 to construct $n+1$ binary relations instead of an edge-colored graph in $n+1$ colors. Referring to the graph colored in two colors constructed lemma 1 , let $R=\{(x, y): c(x y)=r\}$ and $B=\{(x, y): c(x y)=b\}$. Partition $B$ into $n$ disjoint subsets in the following way: Let $2 \ell$ be the number of asymmetric diversity atoms, so that $b_{1}, \ldots, b_{2 \ell}$ are asymmetric and $b_{2 \ell+1}, \ldots, b_{n}$ are all symmetric. Order the vertices $v_{1}, \ldots, v_{N}$. Let $V_{<}=\left\{\left(v_{i}, v_{j}\right): i<j\right\}$. Assign pairs from $V_{<}$to sets $B_{1}, \ldots, B_{n}$ uniformly at random. Now we assign the remaining pairs $\left(v_{j}, v_{i}\right)$ as follows. For $i, j$ with $i<j$,

(i) if $\left(v_{i}, v_{j}\right) \in B_{m}$ and $m>2 \ell$, then $\left(v_{j}, v_{i}\right) \in B_{m}$;

(ii) if $\left(v_{i}, v_{j}\right) \in B_{m}$ and $1 \leq m \leq \ell$, then $\left(v_{j}, v_{i}\right) \in B_{m+\ell}$;

(iii) if $\left(v_{i}, v_{j}\right) \in B_{m}$ and $\ell<m \leq 2 \ell$, then $\left(v_{j}, v_{i}\right) \in B_{m-\ell}$.

Thus we have $B_{m} \breve{ }=B_{m+\ell}$ for $m \leq \ell$. Then by making superficial changes to the remainder of the proof we establish the result for the nonsymmetric case. Thus we have the following: 
Theorem 2. Any finite integral relation algebra with one flexible atom and with all (mandatory) diversity cycles involving the flexible atom is representable on arbitrarily large finite sets.

\section{Acknowledgments}

We thank Maria Axenovich for her valuable suggestions and comments, and the anonymous reviewer for suggesting improvements to the paper. We dedicate this paper to Samantha Alm, who during its writing came into existence.

\section{References}

[1] Stephen D. Comer. Color schemes forbidding monochrome triangles. In Proceedings of the fourteenth Southeastern conference on combinatorics, graph theory and computing (Boca Raton, Fla., 1983), volume 39, pages 231-236, 1983.

[2] Stephen D. Comer. Combinatorial aspects of relations. Algebra Universalis, 18(1):7794, 1984.

[3] P. Jipsen, R. D. Maddux, and Z. Tuza. Small representations of the relation algebra $\mathcal{E}_{n+1}(1,2,3)$. Algebra Universalis, 33(1):136-139, 1995.

[4] J.G. Kalbfleisch. Chromatic Graphs and Ramsey's Theorem. Ph.D. dissertation, University of Waterloo, 1966.

[5] R. C. Lyndon. Relation algebras and projective geometries. Michigan Math. J., $8(1): 21-28,1961$.

[6] Roger D. Maddux. A perspective on the theory of relation algebras. Algebra Universalis, 31(3):456-465, 1994.

[7] Konrad Piwakowski and Stanisław P. Radziszowski. $30 \leq R(3,3,4) \leq 31$. J. Combin. Math. Combin. Comput., 27:135-141, 1998. 